\title{
Optimality Conditions for Discrete Optimal Control Problems
}

\author{
Fang-Di Kong \\ College of Electrical and Information Engineering, Lanzhou University of Technology, Lanzhou, 730050, China \\ Email: kongfd@lut.cn
}

\begin{abstract}
In this paper we consider the optimality conditions for the following discrete optimal control problem:

minimize $J(x, u):=\sum_{k=0}^{N-1} g(k, x(k+1), u(k))$,

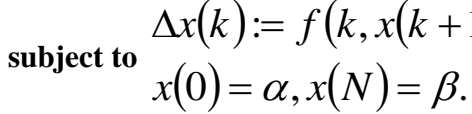

$$
\Delta x(k):=f(k, x(k+1), u(k)), k \in[0, N-1],
$$
\end{abstract}

The necessary and sufficient condition for all feasible solutions satisfying the necessary optimality condition to be optimal solutions of the above problem is established.

Keywords-discrete optimal control problem; optimality condition.

\section{INTRODUCTION}

Throughout this paper, we always assume that $[0, \mathrm{~N}]=\{\mathrm{k} \in \mathrm{Z} \mid 0 \leq \mathrm{k} \leq N\} \quad$ for any $N \in \mathrm{Z} \quad$ with $0 \leq \mathrm{N}-2$ and the vectors are column vectors. By $q$ we denote the zero vector of the corresponding size.

The study of optimality conditions is an important topic in optimization. It is the well-known necessary condition. Generally speaking, the functions satisfying the necessary condition are not necessarily optimal solutions. Recently, the study of sufficient optimality conditions of the optimal control problems has received much attention from many authors (see for example [1,2] and the references therein). In the same time, the study of the optimality conditions of discrete optimal control problems is also of interest to many researchers (see, for example [3-8], and the references therein).

In particular, Arana-Jimenez, Osuna-Gomez, RuizGarzon and Rojas-Medar [2] obtained a characterization of optimal solutions of the optimal control problem:

$\operatorname{minimize} \int_{a}^{b} f(t, x(t), u(t)) d t$

subject to

$$
\begin{aligned}
& x(a)=\alpha, x(b)=\beta, \\
& g(t, x(t), u(t)) \leq 0, t \in[a, b], \\
& h(t, x(t), u(t))=\dot{x}(t), t \in[a, b] .
\end{aligned}
$$

In 2000, Hilscher and Zeidan [3] discussed the optimality conditions for following discrete optimal control problem

$$
\begin{aligned}
\operatorname{minimize} & F(x, u):=K\left(x_{N+1}\right)+\sum_{k=0}^{N} g\left(k, x_{k+1}, u_{k}\right) \\
& \Delta x_{k}=f\left(k, x_{k+1}, u_{k}\right), k \in[0, N] \\
& x_{0}=a, \varphi\left(x_{N+1}\right)=0
\end{aligned}
$$

Motivated by the above-mentioned works, in this paper we consider the optimality conditions for the following discrete optimal control problem:

minimize

$$
J(x, u):=\sum_{k=0}^{N-1} g(k, x(k+1), u(k)),
$$

subject to

$$
\begin{aligned}
& \Delta x(k)=f(k, x(k+1), u(k)), k \in[0, N-1], \\
& x(0)=\alpha, x(N)=\beta .
\end{aligned}
$$

where $\quad \alpha, \beta \in R^{n}, x:[0, N] \rightarrow R^{n}, u:[0, N] \rightarrow R^{m}$, $g:[0, N-1] \times R^{n} \times R^{m} \rightarrow R$, $f:[0, N-1] \times R^{n} \times R^{m} \rightarrow R^{n}$ and $g(k, y, r), f(k, y, r)$ has continuous partial derivatives with respect to the components of the vector variables $y$ and $r$ for all $k \in[0, N-1]$.

We will use the notation

$$
\begin{aligned}
& g_{y}(k, y, r)=\left[g_{y_{1}}(k, y, r), g_{y_{2}}(k, y, r), \mathrm{L}, g_{y_{n}}(k, y, r)\right], \\
& g_{r}(k, y, r)=\left[g_{r_{1}}(k, y, r), g_{r_{2}}(k, y, r), \mathrm{L}, g_{r_{m}}(k, y, r)\right], \\
& f_{y}(k, y, r)=\left(\begin{array}{l}
f_{y_{1}}^{1}(k, y, r) f_{y_{2}}^{1}(k, y, r), \cdots, f_{y_{n}}^{1}(k, y, r) \\
f_{y_{1}}^{2}(k, y, r) f_{y_{2}}^{2}(k, y, r), \cdots, f_{y_{n}}^{2}(k, y, r) \\
\cdots \\
f_{y_{1}}^{n}(k, y, r) f_{y_{2}}^{n}(k, y, r), \cdots, f_{y_{n}}^{n}(k, y, r)
\end{array}\right),
\end{aligned}
$$




$$
f_{r}(k, y, r)=\left(\begin{array}{l}
f_{r_{1}}^{1}(k, y, r) f_{r_{2}}^{1}(k, y, r), \cdots, f_{r_{m}}^{1}(k, y, r) \\
f_{r_{1}}^{2}(k, y, r) f_{r_{2}}^{2}(k, y, r), \cdots, f_{r_{m}}^{2}(k, y, r) \\
\cdots \\
f_{r_{1}}^{n}(k, y, r) f_{r_{2}}^{n}(k, y, r), \cdots, f_{r_{m}}^{n}(k, y, r)
\end{array}\right),
$$

to denote the gradients of $g$ and $f$ with respect to $y$ and with respect to $r$ respectively, where $y_{i}(i=1,2, \cdots, n)$ is the $i$ th component of $y \in R^{n}$, similar notation is used for $r_{j}$ and the superscripts denote the components of the $n$ dimensional vector function.

It is worth mentioning that we obtain the necessary and sufficient conditions for all feasible solutions satisfying the necessary optimality condition to be optimal solutions of the problem (1.3)

$$
F=\left\{(x, u) \mid x \in E_{1}, u \in E_{2}, \Delta x(k)=f(k, x(k+1), u(k)), k \in[0, N-1], x(0)=\alpha, x(N)=\beta\right\} .
$$

Lemma 2.1 Let $\varphi:[0, N] \rightarrow R^{n}$. If

$$
\sum_{k=0}^{N-1} \varphi^{T}(k) \eta(k+1)=0
$$

for all $\eta:[0, N] \rightarrow R^{n}$ with $\eta(0)=\eta(N)=0$, then

$$
\varphi(\mathrm{k})=0, k \in[0, N-2] \text {. }
$$

Proof. Fix $t \in[0, N-2]$ and fix $i \in\{1,2, \cdots, n\}$. Define $\eta_{t}=[0, N] \rightarrow R^{n}$ by

$$
\eta_{t}(k)=\left\{\begin{array}{l}
e_{i,} k=t+1, \\
0, \text { otherwise } .
\end{array}\right.
$$

where $e_{i}$ is the unit vector of $R^{n}$ in the $i$-th direction. Since $\eta_{t}(0)=\eta_{t}(N)=0$, we get from (2.1) with $\eta$ replaced by $\eta_{t}$ that

$$
\varphi^{T}(t) e_{i}=0
$$

Since this is true for each $i, 1 \leq i \leq n$, we have

$$
\varphi(t)=0
$$

As $t \in[0, N-2]$ is arbitrary, we get

$$
\varphi(k)=0, k \in[0, N-2] \text {. }
$$

\section{THE OPTIMALITY CONDITION OF DISCRETE OPTIMAL CONTROL PROBLEMS}

In this section we consider the optimality condition for the discrete optimal control problem (1.3).

Let

$$
\mathrm{E}_{1}=\left\{x \mid x:[0, N] \rightarrow R^{n}\right\}, \mathrm{E}_{2}=\left\{u \mid u:[0, N] \rightarrow R^{m}\right\},
$$

be equipped with the norm

$$
\|x\|=\max _{k \in[0, N]}\left|x(k),,\|u\|=\max _{k \in[0, N]}\right| u(k) \mid,
$$

where $\|$ is some norm on $R^{n}$ and $R^{m}$.

We denote by $F$ the set of feasible solutions of the discrete optimal control problem (1.3), i.e.,
Lemma 2.2 Let $\varphi, \psi:[0, N] \rightarrow R^{n}$. If

$$
\sum_{k=0}^{N-1}\left[\varphi^{T}(k) \eta(k+1)+\psi^{T}(k) \Delta \eta(k)\right]=0
$$

for all $\eta:[0, N] \rightarrow R^{n}$ with $\eta(0)=\eta(N)=0$, then

$$
\varphi(k)=\Delta \psi(k), k \in[0, N-2] .
$$

Proof. Using the summation by parts formula

$$
\sum_{k=0}^{N-1} u^{T}(k) \Delta v(k)=\left.u^{T}(k) v(k)\right|_{0} ^{N}-\sum_{k=0}^{N-1}\left[\Delta u^{T}(k)\right] v(k+1)
$$

on the second term under the sum we obtain

$$
\begin{aligned}
& \sum_{k=0}^{N-1}\left[\varphi^{T}(k) \eta(k+1)+\psi^{T}(k) \Delta \eta(k)\right] \\
& =\sum_{k=0}^{N-1}\left\{\varphi^{T}(k)-\left[\Delta \psi^{\mathrm{T}}(k)\right]\right\} \eta(k+1)+\left.\psi^{T}(k) \eta(k)\right|_{0} ^{\mathrm{N}} \\
& =\sum_{k=0}^{N-1}\left\{\varphi^{T}(k)-\left[\Delta \psi^{\mathrm{T}}(k)\right]\right\} \eta(k+1) \\
& =0
\end{aligned}
$$

for all $\eta:[0, N] \rightarrow R^{n}$ with $\eta(0)=\eta(N)=0$.

By Lemma 2.1, we have

$\varphi(k)-\Delta \psi(k)=0, k \in[0, N-2]$,

i.e. 
Definition $2.1([3-5])(\hat{x}, \hat{u}) \in F$ is called a local minimum of the discrete optimal control problem (1.3) provided there exists $\delta>0$ such that $J(\hat{x}, \hat{u}) \leq J(x, u)$ for all $(x, u) \in F$ with $\|x-\hat{x}\|<\delta$ and $\|u-\hat{u}\|<\delta$.

Definition 2.2 $(\hat{x}, \hat{u}) \in F$ is said to be an optimal solution of the discrete optimal control problem (1.3) if

$$
\begin{aligned}
J(\hat{x}, \hat{u}) \leq J(x, u) \\
g_{y}^{T}(k, \hat{x}(k+1), \hat{u}(k))+f_{y}^{T}(k, \hat{x}(k+1) \hat{u}(k)) \hat{\lambda}(k+1)=-\Delta \hat{\lambda}(k+1), k \in[0, N-2] \\
\quad g_{r}^{T}(k, \hat{x}(k+1), \hat{u}(k))+f_{r}^{T}(k, \hat{x}(k+1), \hat{u}(k)) \hat{\lambda}(k+1)=0, k \in[0, N-2]
\end{aligned}
$$

Theorem 2.4 All feasible solutions satisfying (2.3) and (2.4) in Lemma 2.3 are optimal solutions of the discrete optimal control problem (1.3) if and only if there exists vector functions $\quad \eta:[0, N] \times R^{n} \times R^{m} \times R^{n} \times R^{m} \times R^{n} \rightarrow R^{n}$ and $\xi:[0, N] \times R^{n} \times R^{m} \times R^{n} \times R^{m} \times R^{n} \rightarrow R^{m}$,

such that for all $(x, u),\left(\begin{array}{c}-- \\ x, u\end{array}\right) \in F \quad$ and $\quad$ all $\lambda:[0, N] \rightarrow R^{n}$, the following conditions are fulfilled :

$(H 1)$

$$
\begin{aligned}
& \eta(0, x(0), u(0), \bar{x}(0), \bar{u}(0), \lambda(0))=\eta(N, x(N), u(N), \bar{x}(N), \bar{u}(N), \lambda(N))=0 \\
& \xi(0, x(0), u(0), \bar{x}(0), \bar{u}(0), \lambda(0))=\xi(N, x(N), u(N), \bar{x}(N), \bar{u}(N), \lambda(N))=0
\end{aligned}
$$

$(H 2)$ if $J(x, u)-J(\bar{x}, \bar{u})<0$, then

$$
\begin{aligned}
& \sum_{k=0}^{\mathrm{N}-1}\left\{\begin{array}{l}
{\left[g_{y}(k, \bar{x}(k+1), \bar{u}(k))+\lambda^{T}(k+1) f_{y}(k, \bar{x}(k+1), \bar{u}(k))\right] \eta(k+1, x(k+1), u(k+1), \bar{x}(k+1), \bar{u}(k+1), \lambda(k+1))} \\
-\lambda^{T}(k+1)[\Delta \eta(k, x(k), u(k), \bar{x}(k), \bar{u}(k), \lambda(k))] \\
\left.+\left[g_{r}(k, \bar{x}(k+1), \bar{u}(k))+\lambda^{T}(k+1) f_{r}(k, \bar{x}(k+1), \bar{u}(k))\right] \xi(k+1, x(k+1), u(k+1), \bar{x}(k+1), \bar{u}(k+1), \lambda(k+1))\right)
\end{array}\right\} \\
& \quad \neq 0
\end{aligned}
$$


Proof. $\quad(\Leftarrow)$ Assume that $(\hat{x}, \hat{u}) \in F \quad$ with $\quad(x, u) \in F$, by (2.2), (2.3), (2.4) and $\left(\mathrm{H}_{1}\right)$, we have $(\hat{x}(\cdot), \hat{u}(\cdot), \hat{\lambda}(\cdot))$ satisfying $(2.3)$ and $(2.4)$. Then for all

$$
\begin{aligned}
& \int\left[g_{y}(k, \hat{x}(k+1), \hat{u}(k))+\hat{\lambda}^{T}(k+1) f_{y}(k, \hat{x}(k+1), \hat{u}(k))\right] \eta(k+1, x(k+1), u(k+1), \hat{x}(k+1), \hat{u}(k+1), \hat{\lambda}(k+1)) \\
& \sum_{k=0}^{\mathrm{N}-1}\left\{-\hat{\lambda}^{T}(k+1)[\Delta \eta(k, x(k), u(k), \hat{x}(k), \hat{u}(k), \hat{\lambda}(k))]\right. \\
& \left.+\left[g_{r}(k, \hat{x}(k+1), \hat{u}(k))+\hat{\lambda}^{T}(k+1) f_{r}(k, \hat{x}(k+1), \hat{u}(k))\right] \xi(k+1, x(k+1), u(k+1), \hat{x}(k+1), \hat{u}(k+1), \hat{\lambda}(k+1))\right] \\
& =\sum_{k=0}^{\mathrm{N}-2}\left\{\begin{array}{l}
{\left[\begin{array}{l}
\left.g_{y}(k, \hat{x}(k+1), \hat{u}(k))+\hat{\lambda}^{T}(k+1) f_{y}(k, \hat{x}(k+1), \hat{u}(k))\right] \eta(k+1, x(k+1), u(k+1), \hat{x}(k+1), \hat{u}(k+1), \hat{\lambda}(k+1)) \\
+\Delta \hat{\lambda}^{\mathrm{T}}(k+1)
\end{array}\right]} \\
+\left[g_{r}(k, \hat{x}(k+1), \hat{u}(k))+\hat{\lambda}^{T}(k+1) f_{r}(k, \hat{x}(k+1), \hat{u}(k))\right] \xi(k+1, x(k+1), u(k+1), \hat{x}(k+1), \hat{u}(k+1), \hat{\lambda}(k+1))
\end{array}\right\} \\
& -\left.\hat{\lambda}^{\mathrm{T}}(k+1) \eta(k, x(k), u(k+1), \hat{x}(k), \hat{u}(k), \hat{\lambda}(k))\right|_{0} ^{N-1} \\
& +\left[g_{y}(N-1, \hat{x}(N), \hat{u}(N-1))+\hat{\lambda}^{T}(N) f_{y}(N-1, \hat{x}(N-1), \hat{u}(N-1))\right] \eta(N, x(N), u(N), \hat{x}(N), \hat{u}(N), \hat{\lambda}(N)) \\
& -\hat{\lambda}^{\mathrm{T}}(N) \Delta \eta(N-1, x(N-1), u(N-1), \hat{x}(N-1), \hat{u}(N-1), \hat{\lambda}(N-1)) \\
& +\left[g_{r}(N-1, \hat{x}(N), \hat{u}(N-1))+\hat{\lambda}^{T}(N) f_{r}(N-1, \hat{x}(N), \hat{u}(N))\right] \xi(N, x(N), u(N), \hat{x}(N), \hat{u}(N), \hat{\lambda}(N)) \\
& =-\hat{\lambda}^{\mathrm{T}}(N) \eta(N-1, x(N-1), u(N-1), \hat{x}(N-1), \hat{u}(N-1), \hat{\lambda}(N-1)) \\
& -\hat{\lambda}^{\mathrm{T}}(N) \eta(N, x(N), u(N), \hat{x}(N), \hat{u}(N), \hat{\lambda}(N)) \\
& +\hat{\lambda}^{\mathrm{T}}(N) \eta(N-1, x(N-1), u(N-1), \hat{x}(N-1), \hat{u}(N-1), \hat{\lambda}(N-1))=0
\end{aligned}
$$

Now (2.5) together with $\left(H_{2}\right)$, we get

$$
J(x, u)-J(\hat{x}, \hat{u}) \geq 0 \text { for all }(x, u) \in F
$$

Therefore, $(\hat{x}, \hat{u})$ is an optimal solution of the optimal control problem (1.3).
$(\Rightarrow) \quad$ Let $\quad$ all $\quad\left(\begin{array}{c}-- \\ x, u\end{array}\right) \in F \quad$ with $(\bar{x}(\cdot), \bar{u}(\cdot), \bar{\lambda}(\cdot))$ satisfying (2.3) and (2.4) be optimal solutions of the discrete optimal control problem (1.3). Then we have to find vector functions 
$\eta:[0, N] \times R^{n} \times R^{m} \times R^{n} \times R^{m} \times R^{n} \rightarrow R^{n} \quad$ and $\xi:[0, N] \times R^{n} \times R^{m} \times R^{n} \times R^{m} \times R^{n} \rightarrow R^{m} \quad$, such that $\left(\mathrm{H}_{1}\right)$ and $\left(\mathrm{H}_{2}\right)$ are fulfilled.

Obviously, there exist some vector functions $\eta:[0, N] \times R^{n} \times R^{m} \times R^{n} \times R^{m} \times R^{n} \rightarrow R^{n} \quad$ and $\xi:[0, N] \times R^{n} \times R^{m} \times R^{n} \times R^{m} \times R^{n} \rightarrow R^{m} \quad$, such that $\left(\mathrm{H}_{1}\right)$ is satisfied.

Next, we prove there exists a vector function $\eta:[0, N] \times R^{n} \times R^{m} \times R^{n} \times R^{m} \times R^{n} \rightarrow R^{n} \quad$ and $\xi:[0, N] \times R^{n} \times R^{m} \times R^{n} \times R^{m} \times R^{n} \rightarrow R^{m}$ for all $(\mathrm{x}, u),(\bar{x}, \bar{u}) \in F \quad$ satisfying $\left(H_{1}\right)$, such that $\left(H_{2}\right)$ is fulfilled.

Suppose on the contrary that any vector function

$$
\begin{aligned}
& \eta:[0, N] \times R^{n} \times R^{m} \times R^{n} \times R^{m} \times R^{n} \rightarrow R^{n}, \\
& \xi:[0, N] \times R^{n} \times R^{m} \times R^{n} \times R^{m} \times R^{n} \rightarrow R^{m}, \\
& \eta(0, x(0), u(0), \bar{x}(0), \bar{u}(0), \lambda(0))=\eta(N, x(N), u(N), \bar{x}(N), \bar{u}(N), \lambda(N))=0,
\end{aligned}
$$

$\xi(\cdot, x(\cdot), u(\cdot), \bar{x}(\cdot), \bar{u}(\cdot), \lambda(\cdot)):[0, N] \rightarrow R^{m}$ with

$$
\xi(0, x(0), u(0), \bar{x}(0), \bar{u}(0), \lambda(0))=\xi(N, x(N), u(N), \bar{x}(N), \bar{u}(N), \lambda(N))=0
$$

such that

$$
\begin{gathered}
J(x, u)-J(\bar{x}, \bar{u})<0, \quad \text { but } \\
\sum_{k=0}^{N-1}\left\{\begin{array}{l}
{\left[g_{y}(k, \bar{x}(k+1), \bar{u}(k))+\lambda^{T}(k+1) f_{y}(k, \bar{x}(k+1), \bar{u}(k))\right] \eta(k+1, x(k+1), u(k+1), \bar{x}(k+1), \bar{u}(k+1), \lambda(k+1))} \\
-\lambda^{T}(k+1)[\Delta \eta(k, x(k), u(k), \bar{x}(k), \bar{u}(k), \lambda(k))] \\
+\left[g_{r}(k, \bar{x}(k+1), \bar{u}(k))+\lambda^{T}(k+1) f_{r}(k, \bar{x}(k+1), \bar{u}(k))\right] \xi(k+1, x(k+1), u(k+1), \bar{x}(k+1), \bar{u}(k+1), \lambda(k+1))
\end{array}\right\} \\
\quad \neq 0
\end{gathered}
$$

is not verified. 


$$
\left.\begin{array}{l}
\sum_{k=0}^{\mathrm{N}-1}\left\{\begin{array}{l}
{\left[g_{y}(k, \bar{x}(k+1), \bar{u}(k))+\lambda^{T}(k+1) f_{y}(k, \bar{x}(k+1), \bar{u}(k))\right] \eta(k+1, x(k+1), u(k+1), \bar{x}(k+1), \bar{u}(k+1), \lambda(k+1))} \\
-\lambda^{T}(k+1)[\Delta \eta(k, x(k), u(k), \bar{x}(k), \bar{u}(k), \lambda(k))] \\
+\left[g_{r}(k, \bar{x}(k+1), \bar{u}(k))+\lambda^{T}(k+1) f_{r}(k, \bar{x}(k+1), \bar{u}(k))\right] \xi(k+1, x(k+1), u(k+1), \bar{x}(k+1), \bar{u}(k+1), \lambda(k+1))
\end{array}\right\} \\
\quad=0
\end{array}\right\}
$$

for all $\eta(\cdot, x(\cdot), u(\cdot), \bar{x}(\cdot), \bar{u}(\cdot), \lambda(\cdot)):[0, N] \rightarrow R^{n}$ with

$$
\eta(0, x(0), u(0), \bar{x}(0), \bar{u}(0), \lambda(0))=\eta(N, x(N), u(N), \bar{x}(N), \bar{u}(N), \lambda(N))=0,
$$

and for all $\xi(\cdot, x(\cdot), u(\cdot), \bar{x}(\cdot), \bar{u}(\cdot), \lambda(\cdot)):[0, N] \rightarrow R^{m}$

with

$$
\xi(0, x(0), u(0), \bar{x}(0), \bar{u}(0), \lambda(0))=\xi(N, x(N), u(N), \bar{x}(N), \bar{u}(N), \lambda(N))=0 .
$$

If $\xi(k, x(k), u(k), \bar{x}(k), \bar{u}(k), \lambda(k))=0$ for all

$k \in[0, N]$, then we get

$$
\left.\begin{array}{l}
\sum_{k=0}^{\mathrm{N}-1}\left\{\begin{array}{l}
{\left[g_{y}(k, \bar{x}(k+1), \bar{u}(k))+\lambda^{T}(k+1) f_{y}(k, \bar{x}(k+1), \bar{u}(k))\right] \eta(k+1, x(k+1), u(k+1), \bar{x}(k+1) \bar{u}(k+1), \lambda(k+1))} \\
-\lambda^{T}(k+1)[\Delta \eta(k, x(k), u(k), \bar{x}(k), \bar{u}(k), \lambda(k))]
\end{array}\right. \\
\quad=0
\end{array}\right\}
$$

for all $\eta(\cdot, x(\cdot), u(\cdot), \bar{x}(\cdot), \bar{u}(\cdot), \lambda(\cdot)):[0, N] \rightarrow R^{n}$ with

$$
\eta(0, x(0), u(0), \bar{x}(0), \bar{u}(0), \lambda(0))=\eta(N, x(N), u(N), \bar{x}(N), \bar{u}(N), \lambda(N))=0 .
$$

By Lemma 2.2, we have

$$
g_{y}{ }^{T}(k, \bar{x}(k+1), \bar{u}(k))+f_{y}^{T}(k, \bar{x}(k+1), \bar{u}(k)) \lambda(k+1)=-\Delta \lambda(k+1), k \in[0, N-2]
$$

On the other $\quad$ hand, $\quad$ if
$\eta(k, x(k), u(k), \bar{x}(k), \bar{u}(k), \lambda(k))=0 \quad$ for $\quad$ all
$k \in[0, N]$, then we get 


$$
\begin{aligned}
& \sum_{k=0}^{\mathrm{N}-1}\left\{\left[g_{r}(k, \bar{x}(k+1), \bar{u}(k))+\lambda^{T}(k+1) f_{r}(k, \bar{x}(k+1), \bar{u}(k))\right] \eta(k+1, x(k+1), u(k+1), \bar{x}(k+1), \bar{u}(k+1), \lambda(k+1))\right\} \\
& =0
\end{aligned}
$$

for all $\xi(\cdot, x(\cdot), u(\cdot), \bar{x}(\cdot), \bar{u}(\cdot), \lambda(\cdot)):[0, N] \rightarrow R^{m}$ with

$$
\xi(0, x(0), u(0), \bar{x}(0), \bar{u}(0), \lambda(0))=\xi(N, x(N), u(N), \bar{x}(N), \bar{u}(N), \lambda(N))=0 .
$$

By Lemma 2.1, we have

$$
g_{r}{ }^{T}(k, \bar{x}(k+1), \bar{u}(k))+f_{r}^{T}(k, \bar{x}(k+1), \bar{u}(k)) \lambda(k+1)=0, k \in[0, N-2]
$$

This shows that $(\bar{x}(\cdot), \bar{u}(\cdot), \lambda(\cdot))$ verifies (2.3) and (2.4), and then $(\bar{x}, \bar{u})$ is an optimal solution of the discrete optimal control problem (1.3), i.e., $J(x, u)-J(\bar{x}, \bar{u}) \geq 0$, which is a contradiction.

Therefore, there exist vector functions

$$
\eta(\cdot, x(\cdot), u(\cdot), \bar{x}(\cdot), \bar{u}(\cdot), \lambda(\cdot)):[0, N] \rightarrow R^{n}
$$

and

$$
\xi(\cdot, x(\cdot), u(\cdot), \bar{x}(\cdot), \bar{u}(\cdot), \lambda(\cdot)):[0, N] \rightarrow R^{m},
$$

such that $\left(H_{l}\right)$ and $\left(H_{2}\right)$ are fulfilled.

\section{REFERENCES}

[1] B. Mond, I. Smart, Duality and sufficiency in control problems with invexity, Journal of Mathe-matical Analysis and Applications, Vol. 136, no. 1, pp. 325-333, (1988).
[2] M. Arana-Jimenez, R. Osuna-Gomez, A.Rufian-Lizana, G. RuizGarzon, KT-invex control problem, Applied Mathematics and Computation, Vol. 197, no. 2, pp. 489-496, (2008).

[3] R. Hilscher, V. Zeidan, Discrete optimal control: the accessory problem and necessary optimality conditions, Journal of Mathematical Analysis and Applications, Vol. 243, no. 2 , pp. 429452, (2000).

[4] R. Hilscher, V. Zeidan, Discrete optimal control: second order optimality conditions, Journal of Difference Equations and Applications, Vol. 8, no. 10, pp. 875-896, (2002).

[5] R. Hilscher, V. Zeidan, Second order sufficiency criteria for a discrete optimal control problem, Journal of Difference Equations and Applications, Vol. 8, no. 6, pp. 573-602, (2002).

[6] B. Marinkovic, Optimality conditions for discrete calculus of variations problems, Optimization Letters, Vol. 2008, no. 2, pp. 309318,( 2008).

[7] B. Marinkovi, Positivity optimality conditions for discrete optimal control problems with equality and inequality type of constraints, Positivity, Vol. 12, pp. 535-545, (2008).

[8] N. T. Toan, Q. H. Ansari, J.-C. Yao, Second-order necessary optimality conditions for a discrete optimal control problem, Journal of Optimization Theory and Applications, Vol. 165, no. 3, pp. 812836, (2015). 